\title{
Lifestyle Therapy
}

National Cancer Institute

\section{Source}

National Cancer Institute. Lifestyle Therapy. NCI Thesaurus. Code C15900.

Therapy dealing with lifestyle management and includes: behavioral and dietary

modifications, exercise, stress management, and addiction control. This therapy must be used as major adjunct to "standard care" or be applied as alternative treatment to conventional medicine practices. 